Research Article

\title{
Robust Adaptive Output Feedback Control for a Guided Spinning Rocket
}

\author{
Zhongjiao Shi $\mathbb{D}^{1,2}$ Liangyu Zhao $\mathbb{D}^{1,2}$ and Yeqing $\mathrm{Zhu}^{1,2}$ \\ ${ }^{1}$ Beijing Institute of Technology, Beijing 100081, China \\ ${ }^{2}$ Key Laboratory of Dynamics and Control of Flight Vehicle, Ministry of Education, Beijing 100081, China \\ Correspondence should be addressed to Liangyu Zhao; zhaoly@bit.edu.cn
}

Received 25 October 2017; Revised 5 April 2018; Accepted 18 April 2018; Published 14 May 2018

Academic Editor: Kenneth M. Sobel

Copyright (C) 2018 Zhongiiao Shi et al. This is an open access article distributed under the Creative Commons Attribution License, which permits unrestricted use, distribution, and reproduction in any medium, provided the original work is properly cited.

\begin{abstract}
An adaptive autopilot is presented for the pitch and yaw channels of a guided spinning rocket. Firstly, the uncertain dynamic model of a guided spinning rocket is established, which is used to evaluate the performance of the proposed adaptive autopilot. Secondly, a robust adaptive output feedback autopilot containing a baseline component and an adaptive component is designed. The main challenge that needs to be addressed is the determination of a corresponding square and strictly positive real transfer function. A simple design procedure based on linear matrix inequality is proposed that allows the realization of such a transfer function, thereby allowing a globally stable adaptive output feedback law to be generated. Finally, numerical simulations are performed to evaluate the robustness and tracking performance of the proposed robust adaptive autopilot. The simulation results showed that the robust adaptive output autopilot can achieve asymptotic command tracking with significant uncertainty in control effectiveness, moment coefficient, and measurement noise.
\end{abstract}

\section{Introduction}

Traditional artillery ballistic gun-launched munitions cannot satisfy more and more stringent performance requirements about precision-strike capability, dispersion error reduction, and range augmentation required on modern battlefields. Complex guided systems such as missiles can meet these requirements, but they remain expensive due to the integration of high-quality actuators and sensors. The idea is to develop a guided rocket, which permits to reach a compromise between the low cost of ballistic projectiles and the necessity to have high-performance systems with efficient control algorithms. The guided rocket chosen in this work is a canard-controlled spinning configuration, which makes use of an existing multiple launch rocket system (MLRS). This one has the advantage of simplifying the structure of the control system, avoiding asymmetric ablation, relaxing the manufacturing error tolerance, and improving the penetration ability. It is also easy to implement and does not require the design of a new launch system. However, this kind of configuration has the disadvantages of cross coupling due to the spinning airframe. Besides, uncertainties in control effectiveness and moment coefficients are among the practical challenges in the control system design. Finally, the sensors are of limited performance, due to the low cost and small size specification.

These disadvantages can be handled by developing a flight autopilot using modern multivariable control methods, such as robust control $[1,2]$ and gainscheduling control $[3,4]$. For the dual-channel-controlled spinning rocket, various autopilots were designed, such as rate loop autopilot [5], attitude autopilot [6], acceleration autopilot [7], and three-loop autopilot [8]. However, these related works were carried out under the nominal condition without considering uncertainties which may be experienced during the whole flight trajectory. Additionally, it is difficult to design an autopilot for a guided spinning rocket with excellent performance using the traditional separate channel design method.

Adaptive control is known as a proper method to deal with uncertainties and has been used in numerous 


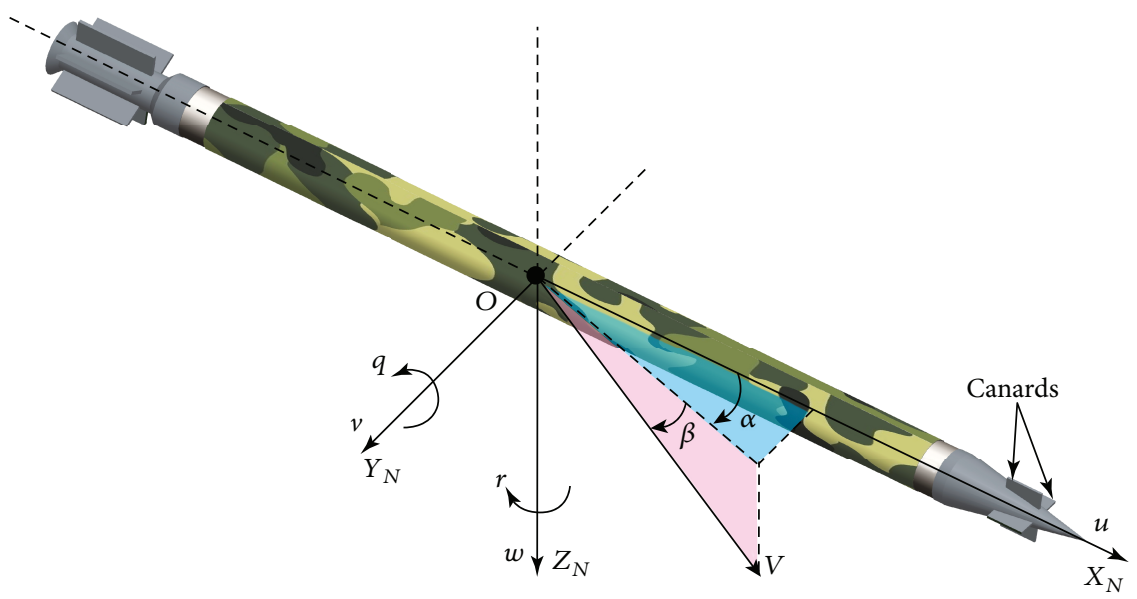

FIGURE 1: Sketch of guided spinning projectile.

applications. Therefore, this is aimed at investigating the potential of robust adaptive control for improving stability and performance of the spinning rocket autopilot. A generic transport aircraft autopilot was designed by employing a modified MRAC scheme to guarantee the transient performance [9]. A Lyapunov-based model reference adaptive PD/PID controller for Satellite Launch Vehicle (SLV) systems was proposed to improve the tracking performance and robustness under wind disturbances [10]. An adaptive integral feedback controller for pitch and yaw channels of an autonomous underwater vehicle (AUV) was designed to handle the actuator saturations [11]. These controllers require that the system state must be measurable [12], which may not always be possible. For this reason, there has been an increasing motivation to develop an adaptive output feedback controller. Existing classical methods of multi-input and multi-output (MIMO) output feedback adaptive control are applicable for square systems; that is, the plant has the same number of inputs and outputs $[13,14]$. Recently, an output feedback adaptive spinning rocket autopilot was designed, but only the acceleration information was used to construct the autopilot [15]. In order to make full use of all the measurable information, the output feedback adaptive autopilot for a spinning rocket with four measurable outputs and two control inputs, which is a nonsquare system, needs to be designed. The main challenge is to make the closedloop transfer functions satisfy the strictly positive real (SPR) property or guarantee the strict passivity of the closed-loop system. These two properties were proved to be equivalent for a linear time-invariant system [16]. For the square system, necessary and sufficient conditions for passifiability of the linear system by output feedback were presented in [17, 18]. An observer-based method was also included in the design of controllers to guarantee the that closed-loop system is SPR [19]. For the nonsquare system, the squaring-down procedure was employed for passification design [20]. And necessary and sufficient conditions for passifiability of nonsquare systems by output feedback were given in [21].

The main contribution of this paper is to combine the observer-based method and squaring-down method to design an adaptive output feedback autopilot for guided spinning rockets. First, a mixing matrix $M$ is designed to make the modified error dynamic model a square system. Then, a Luenberger observer which also serves as the reference model is employed to make the error dynamic model satisfy the SPR property.

The remainder of this paper is structured in the following manner. Section 2 develops the uncertain dynamic model of a dual-channel-controlled spinning rocket. Section 3 presents the robust adaptive output-feedback autopilot design using robust SPR lemma. Section 4 demonstrates the performance of the robust adaptive output-feedback autopilot via numerical simulations. Finally, Section 5 concludes this paper.

\section{Mathematical Model}

The guided spinning rocket considered in this paper is an axially symmetric rolling airframe, as is shown in Figure 1. Two pairs of canard rotating with the airframe are employed as control surfaces twisting and steering the rocket. The maneuver of the airframe requires an autopilot that is able to track different kinds of signals under a different dynamic environment, with slight or even no change of the autopilot architecture.

2.1. Dynamical Equation. According to Newton's second law, the translational motion of center of gravity (cg) can be described in the nonrolling body coordinate system as follows [22]:

$$
m \frac{d \mathbf{V}_{A}}{d t}=m\left(\frac{\partial \mathbf{V}_{N}}{\partial t}+\omega_{r} \times \mathbf{V}_{N}\right)=\mathbf{F}+m \mathbf{g}
$$

where $m$ is the mass of the spinning rocket, $\mathbf{V}_{A}=(x, y, z)^{T}$ is the velocity of $\mathrm{cg}$ represented in inertial coordinate, $\mathbf{V}_{N}=(u, v, w)^{T}$ is the velocity of cg in the nonrolling body coordinate, and $\omega_{r}$ is the angular rate of the nonrolling body coordinate system with respect to the inertial coordinate system. $\mathbf{F}$ is the aerodynamic force, and $\mathbf{g}$ is the acceleration of 
gravity, which are all represented in the nonrolling body coordinate system. All the variables in (1) can be modeled as

$$
\begin{aligned}
\omega_{r} & =(-r \tan \theta, q, r)^{T}, \\
\mathbf{F} & =\left[\begin{array}{c}
F_{x} \\
F_{y} \\
F_{z}
\end{array}\right]=Q S\left[\begin{array}{c}
-C_{x} \\
-C_{N \beta} \beta+C_{N \delta} \delta_{z} \\
-C_{N \alpha} \alpha-C_{N \delta} \delta_{y}
\end{array}\right], \\
\mathbf{g} & =\left[\begin{array}{c}
g_{x} \\
g_{y} \\
g_{z}
\end{array}\right]=\left[\begin{array}{c}
-g \sin \theta \\
-0 \\
g \cos \theta
\end{array}\right],
\end{aligned}
$$

where $q$ and $r$ are angular rates along $O Y_{N}$ and $O Z_{N}$ defined in Figure 1; $\theta$ is the pitch angle of the spinning rocket; $Q$ is the dynamic pressure; $S$ is the reference area; $C_{x}$ is the drag coefficient slope; $C_{N \alpha}$ and $C_{N \beta}$ are the normal force coefficient slopes, due to the character of symmetric $C_{N \alpha}=C_{N \beta}$, so as other coefficients related with $\alpha$ and $\beta ; C_{N \delta}$ is the control force coefficient slope; $\delta_{y}$ and $\delta_{z}$ represent the actuator deflection angles in the nonrolling coordinate system; and $\alpha$ and $\beta$ are the angle of attack and the sideslip angle, respectively. The expressions of $\alpha$ and $\beta$ are presented as

$$
\begin{aligned}
& \alpha=\arctan \left(\frac{w}{u}\right) \approx \frac{w}{V}, \\
& \beta=\arcsin \left(\frac{v}{u}\right) \approx \frac{v}{V} .
\end{aligned}
$$

By substituting (2), (3), and (4) into (1), the force equations are obtained:

$$
\begin{aligned}
\dot{u}+w q-v r & =\frac{F_{x}}{m}+g_{x}, \\
\dot{v}+u r-w r \tan \theta & =\frac{F_{y}}{m}+g_{y}, \\
\dot{w}-u q-v r \tan \theta & =\frac{F_{z}}{m}+g_{z} .
\end{aligned}
$$

According to the theorem of angular momentum, the rotational motion of the airframe can be described in the nonrolling body coordinate system as

$$
\frac{d \mathbf{H}}{d t}=\frac{\partial \mathbf{H}}{\partial t}+\omega_{r} \times \mathbf{H}=\mathbf{M},
$$

where $\mathbf{H}$ is the angular momentum and $\mathbf{M}$ is the moment acting on the rocket. All the variables in (7) are described in the nonrolling body coordinate system as

$$
\begin{aligned}
\mathbf{H} & =\left(I_{x} p, I_{y} q, I_{z} r\right)^{T}, \\
\frac{\partial \mathbf{H}}{\partial t} & =\left(I_{x} \dot{p}, I_{y} \dot{q}, I_{z} \dot{r}\right)^{T},
\end{aligned}
$$

where $I_{x}, I_{y}$, and $I_{z}$ are moments of inertia, due to the character of symmetric $I_{y}=I_{z} ; p$ is the spinning rate in the nonrolling coordinate.
For a canard-controlled spinning rocket, positive $\delta_{z}$ and $\delta_{y}$ create positive moments in $M_{z}$ and $M_{y}$, respectively. Therefore, the aerodynamic moment can be expressed as

$$
M=\left[\begin{array}{c}
M_{x} \\
M_{y} \\
M_{z}
\end{array}\right]=Q S l\left[\begin{array}{c}
C_{l \delta x} \delta_{x}-C_{l p} \frac{p l}{V} \\
C_{m \alpha} \alpha-C_{m q} \frac{q l}{V}-C_{m p \alpha} \beta \frac{p l}{V}+C_{m \delta} \delta_{y} \\
-C_{m \alpha} \beta-C_{m q} \frac{r l}{V}-C_{m p \alpha} \alpha \frac{p l}{V}+C_{m \delta} \delta_{z}
\end{array}\right],
$$

where $l$ is the reference length; $C_{l \delta x}$ is the rolling moment coefficient due to the canted angle of tails, which is denoted as $\delta_{x} ; C_{l p}$ is the rolling damping moment coefficient; $C_{m \alpha}$ is the static moment coefficient; $C_{m q}$ is the damping moment coefficient; $C_{m p \alpha}$ is the Magnus moment coefficient; and $C_{m \delta}$ is the control moment coefficient.

Remark 1. The guided spinning rocket considered in this paper is a fin-stabilized airframe. For a fin-stabilized rocket, the classic Magnus force is typically ignored in (3) since its effect is rather small for slowly rolling rockets. However, the Magnus moment with physical mechanisms specific to fin-stabilized rockets cannot be ignored in (10), which can be expressed as a dynamic side moment due to spin rate $p$ and angle of attack $\alpha$ or sideslip angel $\beta$ in the form of $C_{m p \alpha} \alpha(p l / V)[23]$.

By substituting (8), (9), and (10) into (7), the moment equations are obtained:

$$
\begin{aligned}
I_{x} \dot{p} & =M_{x}, \\
I_{y} \dot{q}+I_{x} p r+I_{y} r^{2} \tan \theta & =M_{y}, \\
I_{y} \dot{r}-I_{x} p q-I_{y} q r \tan \theta & =M_{z} .
\end{aligned}
$$

Equations (6) and (11) are the nonlinear dynamical model of a spinning rocket, which represents the angular motion of the spinning rocket.

2.2. Linearized Lateral Equation. For flight control which corresponds to the tracking of the dual-canard-controlled spinning rocket acceleration command, only the lateral dynamic system is necessary. In order to simplify the design procedure of the autopilot, some common and reasonable assumptions are made to linearize the lateral dynamic model of the spinning projectile [7]:

Assumption 1. The velocity, roll rate, mass, and aerodynamic coefficients of the rocket remain constant over a short time period.

Assumption 2. For simplicity, the gravity and the small canard force are ignored. The lateral velocities $v$ and $w$ are small with respect to the axis velocity $u$, so that $u \approx V$. 
Applying the above assumptions to (6) and (11), the linearized angular motion of the spinning rocket can be described as

$$
\begin{aligned}
& \dot{\beta}=-r-c_{N \alpha} \beta, \\
& \dot{\alpha}=q-c_{N \alpha} \alpha, \\
& \dot{q}=c_{m \alpha} \alpha-c_{m p \alpha} P_{p} \beta-c_{m q} q-P_{p} r+c_{m \delta} \delta_{y}, \\
& \dot{r}=-c_{m \alpha} \beta-c_{m p \alpha} P_{p} \alpha-c_{m q} r-P_{p} q+c_{m \delta} \delta_{z},
\end{aligned}
$$

where the dimensionless coefficients are defined as $c_{N \alpha}=$ $Q S C_{N \alpha} / m V, \quad c_{m \alpha}=Q S l C_{m \alpha} / I_{y}, \quad c_{m q}=Q S l^{2} C_{m q} / I_{y} V, \quad c_{m p \alpha}=$ $Q S l^{2} C_{m p \alpha} / I_{x} V, c_{m \delta}=Q S l C_{m \delta} / I_{y}$, and $P_{p}=p\left(I_{x} / I_{y}\right)$.

Usually, not all the states of a spinning rocket in (12) can be measured by sensors, especially the angle of attack $\alpha$ and the sideslip angle $\beta$. Low-cost inertial measurement units (IMUs) are the general sensors installed in the spinning rocket, which can provide the accelerations $a_{y}, a_{z}$, and angular rates $q$ and $r$ of the lateral motion. In this condition, the accelerations measured by IMU along the $O Y_{N}$ and $O Z_{N}$ axes can be expressed as

$$
\left[\begin{array}{l}
a_{y} \\
a_{z}
\end{array}\right]=\left[\begin{array}{cc}
-c_{N \alpha} V & 0 \\
0 & -c_{N \alpha} V
\end{array}\right]\left[\begin{array}{l}
\beta \\
\alpha
\end{array}\right] .
$$

Hence, the overall dynamic equation of the spinning rocket can be rearranged to the following state-space form,

$$
\begin{aligned}
\dot{x}_{p}(t) & =A_{p} x_{p}(t)+B_{p} u(t), \\
y_{p}(t) & =C_{p} x_{p}(t), \\
z(t) & =C_{z} x_{p}(t),
\end{aligned}
$$

where $x_{p}=\left[\begin{array}{llll}\beta & \alpha & q & r\end{array}\right]^{T} \in \mathbb{R}^{n_{p}}$ is the state vector, $u=$ $\left[\delta_{y} \delta_{z}\right]^{T} \in \mathbb{R}^{m}$ is the control input vector, $y_{p}=$ $\left[\begin{array}{llll}a_{y} & a_{z} & q & r\end{array}\right]^{T} \in \mathbb{R}^{n_{p}}$ is the measurement output vector, and $z=\left[a_{y} a_{z}\right] \in \mathbb{R}^{r}$ is the regulated output vector that is also measured. $A_{p} \in \mathbb{R}^{n_{p} \times n_{p}}$ is the system matrix, $B_{p} \in \mathbb{R}^{n_{p} \times m}$ is the input matrix, $C_{p} \in \mathbb{R}^{p_{p} \times n_{p}}$ is the measurement output matrix, and $C_{z} \in \mathbb{R}^{r \times n_{p}}$ is the regulated output matrix, and they are all known matrices.

2.3. Uncertain Dynamic Equation. The dynamic model presented in (14) is the ideal case where all the matrices are known. In reality, these matrices are unknown and are obtained through various methods. The system matrix $A_{p}$ and output matrices $C_{p}$ and $C_{z}$ can be determined through wind-tunnel tests fairly accurately. In contrast, the input matrix $B_{p}$ may not be accurate, as control inputs are subjected to perturbations in the flight period. First, the orientation of the canards can lead to the dynamical parameters different from the trim condition as represented by the nominal model. This effect can be modeled as an additive term $\Psi_{p}^{T} x_{p}$. Second, canard failure caused by electronic circuit or control surface damage is another effect which may cause the inaccuracy of input matrices. This effect can be modeled as constant matched uncertainty weights $\Lambda$. The modified uncertain dynamic model is given as

$$
\begin{aligned}
\dot{x}_{p}(t) & =A_{p} x_{p}(t)+B_{p}\left(\Lambda u(t)+\Psi_{p}^{T} x_{p}\right), \\
y_{p}(t) & =C_{p} x_{p}(t), \\
z(t) & =C_{z} x_{p}(t),
\end{aligned}
$$

where $\Lambda \in \mathbb{R}^{m \times m}$ and $\Psi_{p} \in \mathbb{R}^{n_{p} \times m}$ are all nonsingular matrices.

\section{Robust Adaptive Autopilot Design}

The fight sequence of guided spinning rockets is decomposed into three flight phases: boost phase, free flight phase, and guided phase. In this section, we mainly consider the guidance phase, during which the autopilot begins to work. In the guided phase, guidance law gives the desired commands which are usually in the form of acceleration, to the autopilot based on the target and rocket's relative motion information. Therefore, designing an acceleration autopilot is more reasonable and effective. The underlying problem is to design an acceleration autopilot that is able to track different kinds of signals under different dynamic environments, with slight or even no change of the autopilot architecture. So, the output feedback adaptive control method is employed to design the acceleration autopilot.

It should be noted that the output feedback adaptive autopilot requires the dynamical system in (15) as the controllable, observable, and minimum phases, which can be satisfied by the canard-controlled spinning rocket. The uncertainty $\Psi_{p}$ is bound with $\left\|\Psi_{p}\right\|<\Omega<\infty$ and $\operatorname{rank}\left(C_{p} B_{p}\right)=m$.

3.1. Addition of Integral Error. Command tracking and disturbance rejection are the main issues to be handled through the integral action $[24,25]$. In this light, consider the regulated output signal tracking error which is defined as follows:

$$
\dot{x}_{e}=z_{c m d}-z
$$

where $z_{c m d}$ is a piecewise continuous command signal. An integral error state is defined as

$$
x_{e}=\int_{0}^{t} \dot{x}_{e} d t=\int_{0}^{t}\left(z_{c m d}-z\right) d t .
$$

Appending (17) to the plant in (15), the augmented open-loop dynamics is given by 


$$
\begin{aligned}
& {\left[\begin{array}{c}
\dot{x}_{p} \\
\dot{x}_{e}
\end{array}\right]=} \underbrace{\left[\begin{array}{cc}
A_{p} & 0_{n_{p} \times r} \\
-C_{z} & 0_{r \times r}
\end{array}\right]}_{A} \underbrace{\left[\begin{array}{c}
x_{p} \\
x_{e}
\end{array}\right]}_{x} \underbrace{\left[\begin{array}{c}
B_{p} \\
0_{r \times m}
\end{array}\right]}_{B}\left(\Lambda u+\Psi_{p}^{T} x_{p}\right) \\
&+\underbrace{\left[\begin{array}{c}
0_{n_{p} \times r} \\
I_{r \times r}
\end{array}\right]}_{B_{c m d}} z_{c m d}, \\
& \underbrace{\left[\begin{array}{c}
y_{p} \\
x_{e}
\end{array}\right]}_{y}=\underbrace{\left[\begin{array}{cc}
C_{p} & 0_{n_{p} \times r} \\
0_{r \times n_{p}} & I_{r \times r}
\end{array}\right]}_{C}\left[\begin{array}{c}
x_{p} \\
x_{e}
\end{array}\right] .
\end{aligned}
$$

Therefore, (18) can be written more compactly as follows:

$$
\begin{aligned}
& \dot{x}=A x+B\left(\Lambda u(t)+\Psi^{T} x\right)+B_{c m d} z_{c m d}, \\
& y=C x
\end{aligned}
$$

where $\Psi=\left[\Psi_{p}^{T} 0_{m \times r}\right]^{T}$ is unknown. Define $n=n_{p}+r$, then $x \in \mathbb{R}^{n}$.

3.2. Control Architecture. Following the design procedure in [25], the controller is divided into two parts: a baseline controller and an adaptive controller. The control input $u$ in (15) is described as

$$
u=u_{b l}+u_{a d}
$$

where $u_{a d}$ is the adaptive component and $u_{b l}$ is the baseline component, defined as

$$
u_{b l}=K_{x}^{T} x_{m}
$$

where $K_{x}$ is designed by applying the linear quadratic regulator (LQR) technique on the nominal plant model, that is, when $\Lambda=I$ and $\Psi=0 . x_{m}$ is the state of the observer,

$$
\begin{aligned}
& \dot{x}_{m}=A_{m} x_{m}+B_{c m d} z_{c m d}+L\left(y_{m}-y\right), \\
& y_{m}=C x_{m},
\end{aligned}
$$

where $A_{m}=A+B K_{x}^{T}$, and $K_{x}^{T}$ is selected such that $A_{m}$ is Hurwitz.

Remark 2. The observer (22) serves as three purposes:

(1) Luenberger observer. Matrix $L$ is equivalent to an observer gain, for which the purpose is to estimate the state vector $x$ in plant (19). The estimated state vector helps to construct the baseline controller $u_{b l}$.

(2) Reference model. As our ultimate goal is to establish a model reference adaptive controller, (22) serves as a closed-loop reference model (CRM), which has been proved to result in improving transient properties [26-28].
(3) Robust compensator. The output error feedback term $L\left(y_{m}-y\right)$ can be treated as a robust compensator, making the error dynamics satisfy the SPR property.

The adaptive controller $u_{a d}$ is defined as

$$
u_{a d}=\Theta^{T}(t) x_{m},
$$

where $\Theta(t)$ is the estimated uncertain parameter, to be updated by a well-designed update law. Substituting the controller (20) into the augmented plant (19),

$$
\begin{aligned}
& \dot{x}=A x+B\left(\Lambda\left(K_{x}+\Theta(t)\right)^{T}+\Psi^{T} x\right)+B_{c m d} z_{c m d}, \\
& y=C x .
\end{aligned}
$$

If the proposed control architecture can realize accurate command tracking, an ideal uncertain parameter $\Theta^{*}$ must exists and satisfies the following matching condition,

$$
A_{m}=A+B \Psi^{T}+B \Lambda\left(\Theta^{* T}+K_{x}^{T}\right)
$$

with $\Theta^{* T}=\left(\Lambda^{-1}-I\right) K_{x}^{T}-\Lambda^{-1} \Psi^{T}$.

The error dynamic model between the reference model (22) and the closed-loop plant (24) is resorted to accomplish the whole design procedure. The state error $e_{x}=x-x_{m}$ and the estimate parameter error $\Theta^{\sim}(t)=\tilde{\Theta}(t)-\Theta^{*}$ satisfy the dynamics

$$
\begin{aligned}
& \dot{e}_{x}=\left(A+L C+B \Psi^{T}\right) e_{x}+B \Lambda \tilde{\Theta}^{T}(t) x_{m}, \\
& e_{y}=C e_{x},
\end{aligned}
$$

where $e_{y}$ is the measured output error. The problem of finding a stabilizing adaptive controller is equivalent to finding an observer gain $L$ and an adaptive law for $\Theta(t)$ in (26), so that the underlying transfer function matrix is SPR.

The following adaptive law is employed to update the estimated uncertain parameter,

$$
\dot{\Theta}(t)=-\Gamma x_{m} e_{y}^{T} M^{T} \operatorname{sgn}(\Lambda),
$$

where $\Gamma$, the adaptive gain, is a positive diagonal free design matrix, $M \in \mathbb{R}^{m \times n}$ is a mixing matrix to "square up" the transfer function, and $\operatorname{sgn}(\Lambda)$ represents the sign matrix of the input uncertainty $\Lambda$.

3.3. Robust SPR Design. The SPR property is usually employed to design a stable adaptive law for the error dynamic model (26) of the uncertain plant. However, the definition of SPR is restricted to square transfer functions. As the transfer function of (26) is nonsquare, a suitable mixing matrix $M$ has to be chosen to make the SPR properties applicable to the error dynamic model, yielding

$$
G(s)=M C\left(s I-A-L C-B \Psi^{T}\right)^{-1} B,
$$

where $G(s)$ is a square transfer function of the modified error dynamic model, 


$$
\begin{aligned}
\dot{e}_{x} & =\left(A+L C+B \Psi^{T}\right) e_{x}+B \Lambda \tilde{\Theta}^{T}(t) x_{m}, \\
e_{m} & =M C e_{x}
\end{aligned}
$$

Thus, the design of an output feedback adaptive controller is converted to selecting mixing matrix $M \in \mathbb{R}^{m \times n}$ and observer matrix $L \in \mathbb{R}^{n \times n}$, such that the overall closed-loop system is stable.

The choice of mixing matrix $M$ is not unique. $M$ can be computed as

$$
M=B^{T} P C^{-1},
$$

which satisfies $P B=C^{T} M^{T}$.

Lemma 1. Given the strictly proper transfer matrix $G(s)$ with stabilizable and detectable realization $(A, B, C, D)$, where $A$ $\in \mathbb{R}^{n \times n}$ is asymptotically stable, $B \in \mathbb{R}^{n \times m}, C \in \mathbb{R}^{m \times n}$, and $D$ $\in \mathbb{R}^{m \times m}$; then, $G(s)$ is SPR if, and only if, there exists matrices $P=P^{\top}>0, P \in \mathbb{R}^{n \times n}, Q \in \mathbb{R}^{n \times m}$, and $W \in \mathbb{R}^{m \times m}$ such that

$$
\begin{gathered}
A^{\top} P+P A=-Q Q^{T}, \\
P B-C^{T}=-Q W, \\
D+D^{T}=W^{T} W .
\end{gathered}
$$

Proof 1 . The complete proof on the Lemma 1 can be found in [29] Lemma 3.1.

Lemma 2. Let $H$ and $E$ be given matrices of appropriate dimensions, and $F$ satisfies $F F^{T}<I$; for any $\varepsilon>0$, there is

$$
H F E+(H F E)^{T} \leq \varepsilon H H^{T}+\varepsilon^{-1} E^{T} E .
$$

Proof 2. The complete proof on the Lemma 2 can be found in $[30]$.

The output error feedback term $L\left(y_{m}-y\right)$ can be treated as a robust compensator $u_{m}$ for the error dynamic model, making the error dynamics satisfy the SPR property. First, rewrite the error dynamic model (29) into the canonical form of robust control,

$$
\begin{aligned}
\dot{e}_{x} & =(A+\Delta A) e_{x}+B_{1} w+B_{2} u_{m}, \\
e_{m} & =C_{m} e_{x},
\end{aligned}
$$

where $\triangle A=B \Psi^{T}$ is defined as the uncertain state matrix; $B_{1}=B, \quad B_{2}=I_{n}, \quad C_{m}=M C, \quad w=\Lambda \Theta^{T}(t) x_{m}$, and $u_{m}=L\left(y_{m}-y\right)=L C e_{x}$ are defined as the modified virtual control inputs for modified error dynamics.

The modified error dynamics is SPR and stable if and only if Lemma 1 is satisfied. Equation (29) is strictly proper; that is, $D=0$, so Lemma 1 cannot be used directly. For the case $D=0$, the above set of equations in Lemma 1 reduces to the first two equations with $W=0$ [29]. The observer gain $L$ is designed by finding a statefeedback control of (33), and the following inequality should be satisfied:

$$
(A+\Delta A+L C)^{T} P+P(A+\Delta A+L C)<0,
$$

$$
P B_{1}=C^{T} M^{T} .
$$

$\left[\begin{array}{ccc}X A^{T}+A X+B_{2} W+W^{T} B_{2} & (E X)^{T} & B_{1} \\ * & -\gamma & 0 \\ * & * & -\frac{1}{\gamma}\end{array}\right]<0$,

where $E=\Omega I_{n}$. The matrix in (36) is symmetric, and * represents the transposition of the corresponding item in the matrix.

Lemma 3. Given an uncertain plant (33), the closed-loop system is stable and SPR, if and only if there exist symmetric positive matrices $X$ and $W$ and a constant $\gamma>0$ satisfies

Proof 3. The state matrix uncertainty $\Delta A$ can be described as follows:

$$
\Delta A=B_{1} \Psi^{T}=B_{1} F E,
$$

where $F=\Psi^{T} / \Omega$, and the inequality $F F^{T}<I$ holds.

Define $P=X^{-1}$ and $K=L C=W X^{-1}$, and multiply inequality (36) on both sides by the matrix $\operatorname{diag}(P, I, I)$,

$$
\left[\begin{array}{ccc}
\left(A+B_{2} K\right)^{T} P+\left(A+B_{2} K\right) & E^{T} & P B_{1} \\
* & -\gamma & 0 \\
* & * & -\frac{1}{\gamma}
\end{array}\right]<0 .
$$

By the Schur complement, the LMI defined in (36) is equivalent to the following inequality:

$$
\left(A+B_{2} K\right)^{T} P+P\left(A+B_{2} K\right)+\gamma P B_{1} B_{1}^{T} P+\frac{1}{\gamma} E^{T} E<0 .
$$

With Lemma 2, we have

$$
P B_{1} F E+\left(P B_{1} F E\right)^{T}<\gamma P B_{1} B_{1}^{T} P+\frac{1}{\gamma} E^{T} E .
$$

Thus, inequality (39) is equivalent to the following inequality,

$$
\left(A+B_{2} K+B_{1} F E\right)^{T} P+P\left(A+B_{2} K+B_{1} F E\right)<0,
$$

which means $(A+\Delta A+L C)^{T} P+P(A+\Delta A+L C)<0$. Combining $P B=C^{T} M^{T}$ (34) holds. So, the modified error dynamics is stable and SPR.

The observer gain $L$ can be obtained by solving the feasible LMI with any widely available numerical LMI solver. It can be expressed as

$$
L=W^{*} X^{*-1} C^{-1}
$$

where $W^{*}$ and $X^{*}$ are the feasible solution of (36).

Figure 2 shows the structure of the control loop, where the plant is the dynamical model of the spinning rocket in (14), the reference model is (22), and the adaptive law is (23), 


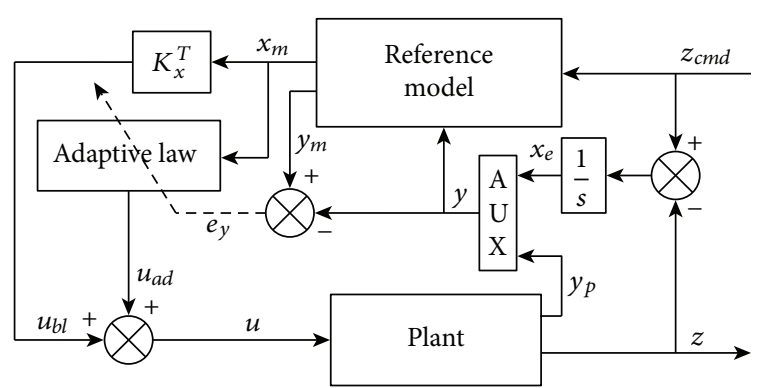

Figure 2: Control architecture.

3.4. Stability Analysis. Given the uncertain linear system in (19), the reference model in (22) with $L$ as in (42), the control architecture in (20), and the update law in (27) result in global stability, with $\lim _{t \rightarrow \infty} e_{x}=0$.

Consider the following Lyapunov function candidate,

$$
V\left(e_{x}, \tilde{\Theta}\right)=e_{x}^{T} P e_{x}+\operatorname{tr}\left(|\Lambda| \tilde{\Theta} \Gamma^{-1} \tilde{\Theta}\right),
$$

where $|\Lambda|$ represents the absolute value of each entry of the matrix element, satisfying $\Lambda=\operatorname{sgn}(\Lambda)|\Lambda|$.

The time derivative of (43) along the system trajectories is given by

$$
\dot{V}=-e_{x}^{T} Q e_{x}+2 e_{x}^{T} P B \Lambda \tilde{\Theta}^{T} x_{m}+2 \operatorname{tr}\left(|\Lambda| \tilde{\Theta}^{T} \Gamma^{-1} \dot{\tilde{\Theta}}\right),
$$

where $\quad\left(A+B \Psi^{T}+L C\right)^{T} P+P\left(A+B \Psi^{T}+L C\right)=-Q<0$. Substituting the update law given in (27) yields

$$
\begin{aligned}
\dot{V} & =-e_{x}^{T} Q e_{x}+2 e_{x}^{T} P B \Lambda \tilde{\Theta}^{T} x_{m}-2 \operatorname{tr}\left(|\Lambda| \tilde{\Theta}^{T} x_{m} e_{y}^{T} M^{T} \operatorname{sgn}(\Lambda)\right) \\
& =-e_{x}^{T} Q e_{x}+2 e_{x}^{T} P B \Lambda \tilde{\Theta}^{T} x_{m}-2 e_{y}^{T} M^{T} \Lambda \tilde{\Theta}^{T} x_{m} \\
& =-e_{x}^{T} Q e_{x}+2 e_{x}^{T} P B \Lambda \tilde{\Theta}^{T} x_{m}-2 e_{x}^{T} C^{T} M^{T} \Lambda \tilde{\Theta}^{T} x_{m} \\
& =-e_{x}^{T} Q e_{x} \leq 0,
\end{aligned}
$$

which implies that $V$ is a Lyapunov function. Since $V>0$ and $\dot{V} \leq 0$, then $V(t) \leq V(0)<\infty$. Thus, $V(t) \in L_{\infty}$, which means $e_{x}, \tilde{\Theta} \in L_{\infty}$. Since $z_{c m d}, e_{x} \in L_{\infty}$, and the reference model are stable, $x_{m} \in L_{\infty}$, which implies that $x_{p} \in L_{\infty}$.

Furthermore, asymptotic stability of the tracking errors is demonstrated by invoking LaSalle's invariance principle, which states that, for a negative semidefinite Lyapunov system in the form of (45), all system trajectories are contained within the domain $\Omega_{0}=\left\{\left[e_{x}, \Theta(t)\right] \mid V\left(\left[e_{x}, \Theta(t)\right], t\right) \leq V\left(\left[e_{x}\right.\right.\right.$, $\Theta(t)], 0)\}$, where the subscript \{\}$_{0}$ denotes the initial conditions, and the entire state space $\left[e_{x}, \Theta(t)\right]$ ultimately reaches the domain $\Omega_{f}=\Omega_{0} \cap \Omega_{z}$, where $\Omega_{z}$ denotes the domain defined by the Lyapunov derivative identical to zero. In other words, the state space ultimately reaches the domain defined by $\dot{V}\left(\left[e_{x}, \Theta(t)\right], t\right) \equiv 0[31,32]$. Because $\dot{V}\left(\left[e_{x}, \Theta(t)\right], t\right)$ is negative-definite in $e_{x}$, the system ends with $e_{x} \equiv 0$. Thus, $x(t) \rightarrow x_{m}(t)$ and the bound reference tracking of $z_{c m d}$ by $z$ follow from the stability of the closed-loop system.
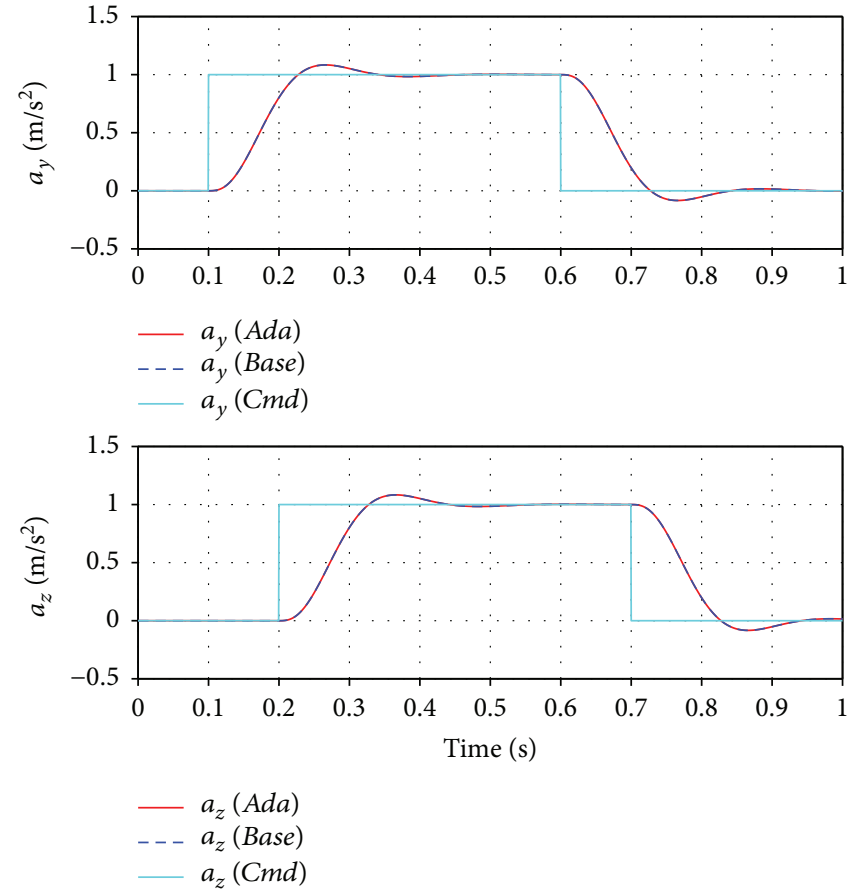

FIgURE 3: The nominal case tracking performance.

Remark 3. In this paper, the adaptive parameter $\Theta(t)$ is not guaranteed to converge to its true unknown value $\Theta^{*}$ nor is it assured to converge to constant value in any way. All that is known is that the unknown parameter remains uniformly bound in time. Sufficient conditions for parameter convergence are known as persistency of excitation.

\section{Case Study}

In this section, numerical examples are performed to evaluate the performance of the output feedback adaptive control scheme applied to the autopilot design for the dual-canard-controlled spinning rocket. All the simulations are performed in MATLAB R2017b with a 64-bit processor, 16 GB memory, and $0.001 \mathrm{~s}$ time-step. Robust Control Toolbox 6.4 in MATLAB is employed to obtain a feasible solution of observer (36). And all the simulations are performed with an adaptive controller acting on the nonlinear model.

The nominal model for autopilot design is the linearized lateral dynamics of the spinning rocket at the speed $V$ of $581 \mathrm{~m} / \mathrm{s}$ and altitude $H$ of $5000 \mathrm{~m}$. The numerical values for the linear system matrices can be found in Appendix A.

All the eigenvalues of the nominal plant system matrix $A_{p}$ have a negative real part, which means the nominal plant is stable.

We show the response of the closed-loop system with the baseline controller and adaptive controller, respectively. Square-wave commands with amplitude of $1 \mathrm{~m} / \mathrm{s}^{2}$ and frequency of $1 \mathrm{~Hz}$ are applied to the pitch and yaw channel; there is a $0.1 \mathrm{~s}$ time lag between the two channels. It is observed in Figure 3 that both the baseline controller and the adaptive controller are able to guide the spinning rocket following the acceleration command and eventually achieved 

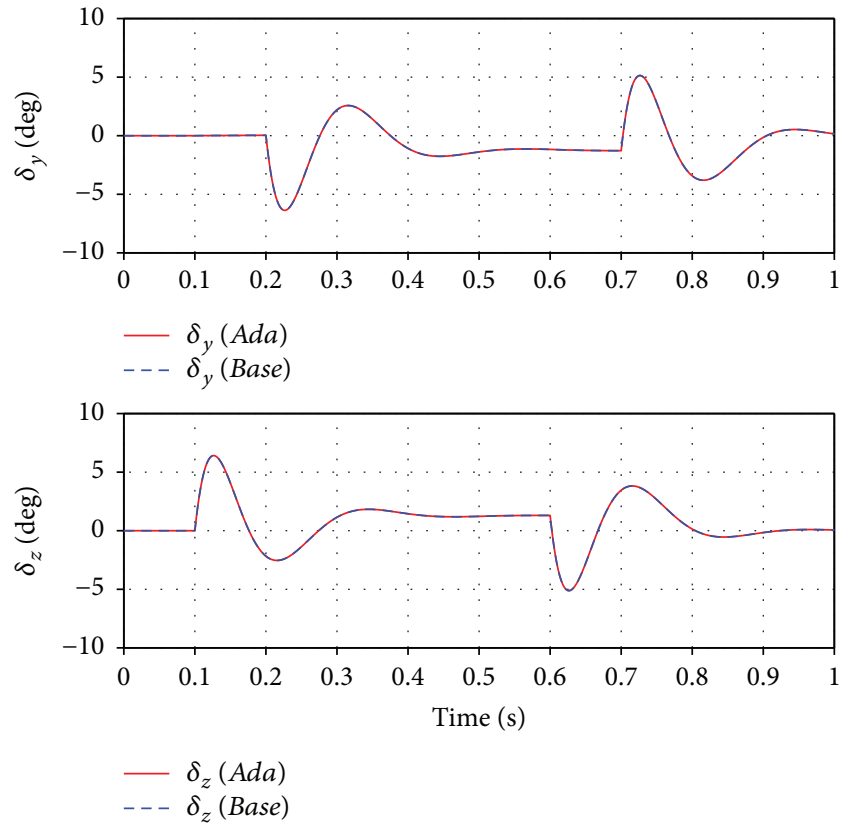

Figure 4: The nominal case control signal.
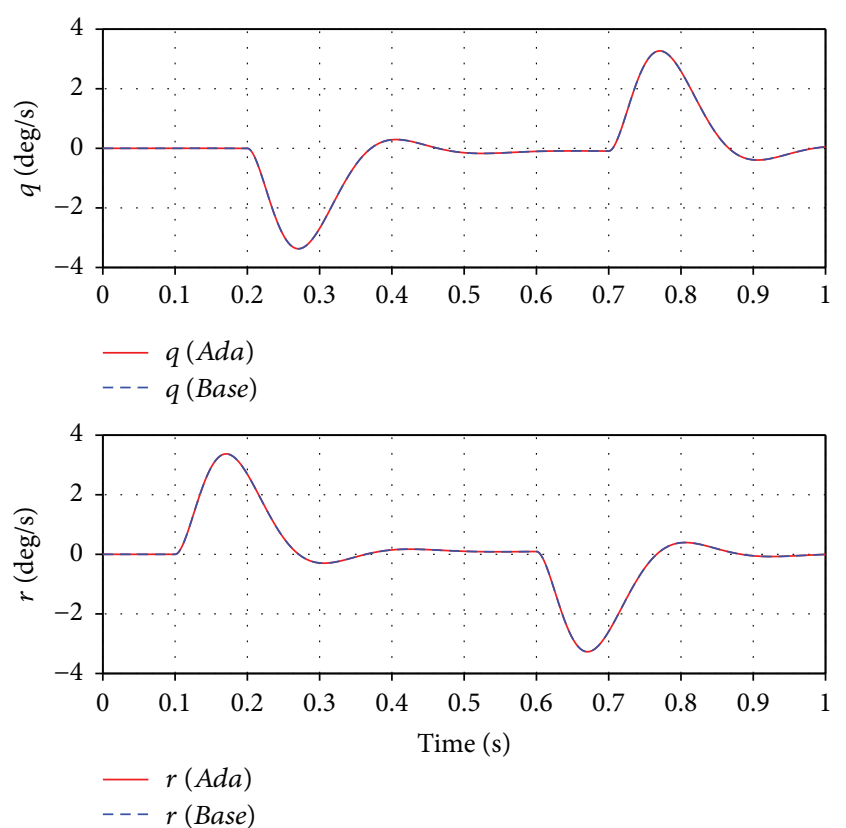

Figure 5: The nominal case angular rates.

a zero tracking error. Figure 4 presents the behavior of control signals in the pitch and yaw channels. Only a small amount of canard deflection angles is used to achieve the command tracking. The angular rates of pitch and yaw channels are presented in Figure 5, which shows a good performance in both channels. Considering simulation results for this case, both the proposed adaptive controller and the baseline controller present a proper performance in the presence of the disturbances enforced due to the channel couplings.
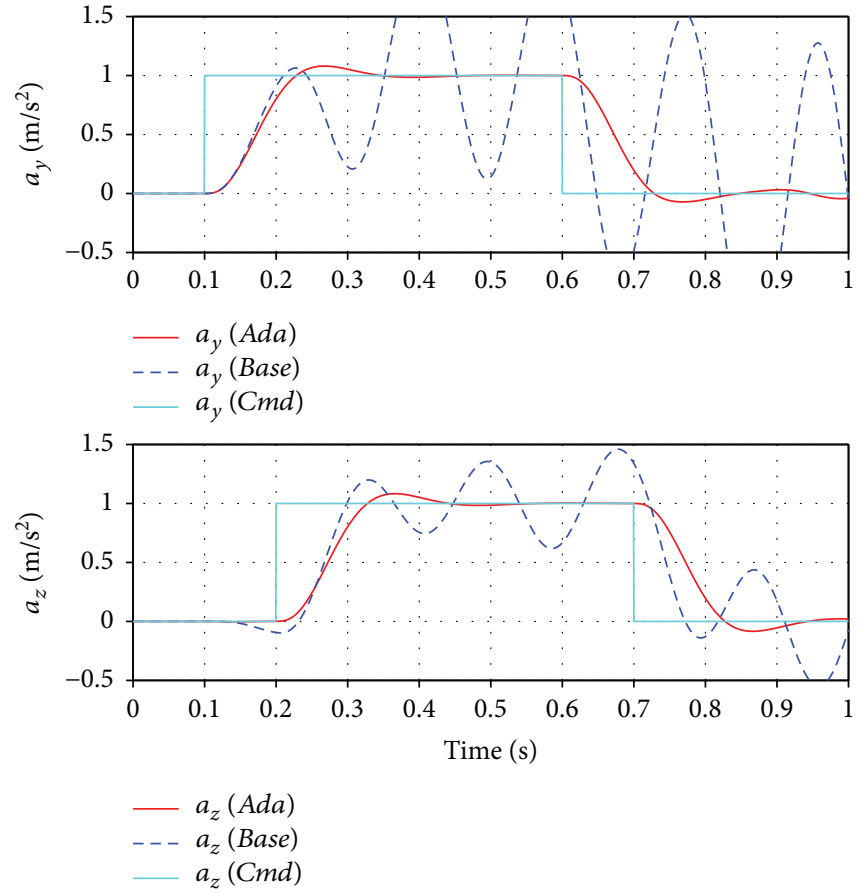

FIgURE 6: The uncertain case tracking performance.
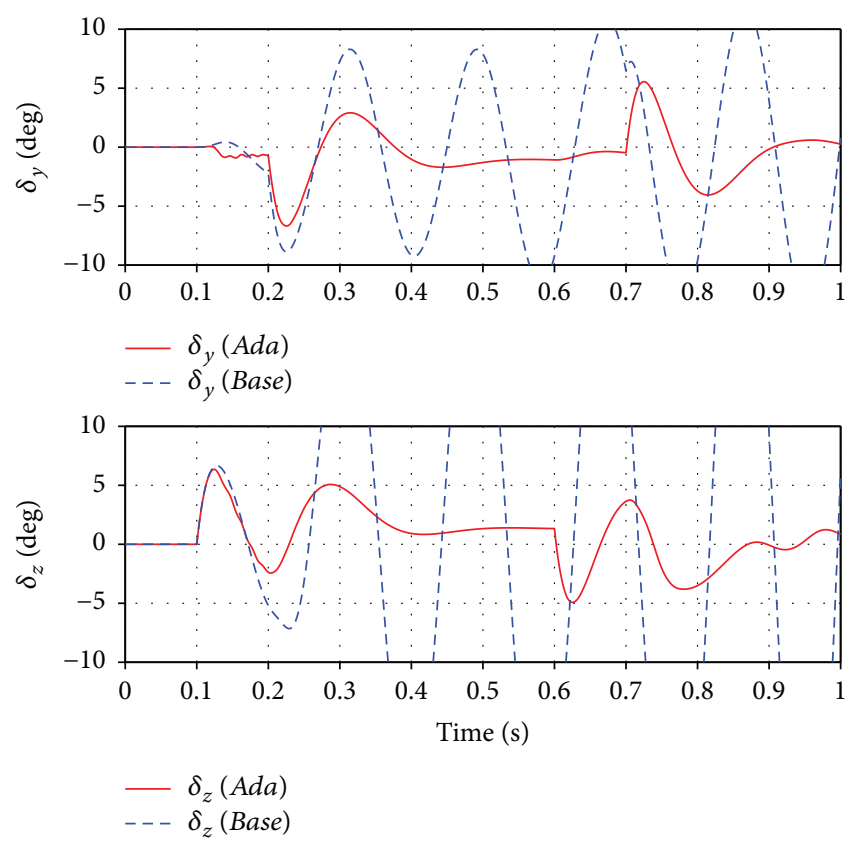

FIgURE 7: The uncertain case control signal.

The same controllers were used in the presence of the following uncertainties:

$$
\Lambda=0.9 I, \quad \Psi=\left[\begin{array}{cccccc}
0 & 0 & 0.12 & 0 & 0 & 0 \\
0 & 0 & 0 & 0.12 & 0 & 0
\end{array}\right] .
$$

Such uncertainties stem from the fact that the model parameters are expected to vary significantly (by about 

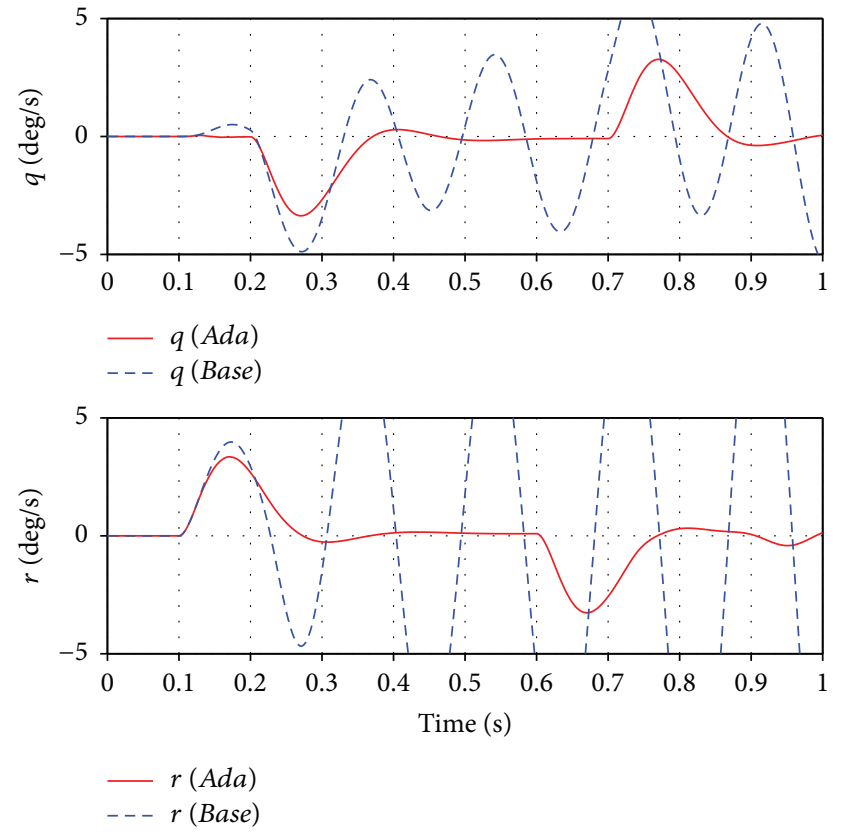

FIGURE 8: The uncertain case angular rates.
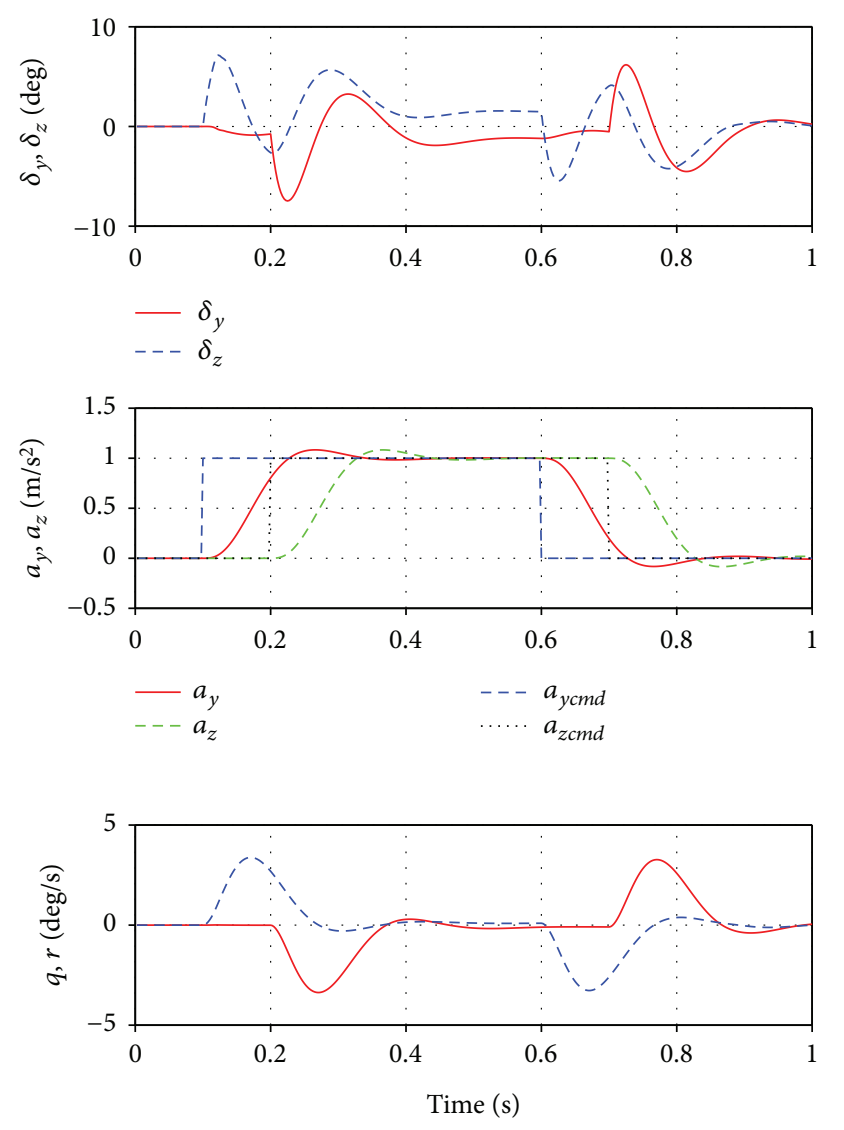

$-q$

FIGURE 9: Control performance with $\Lambda=0.9 I$.
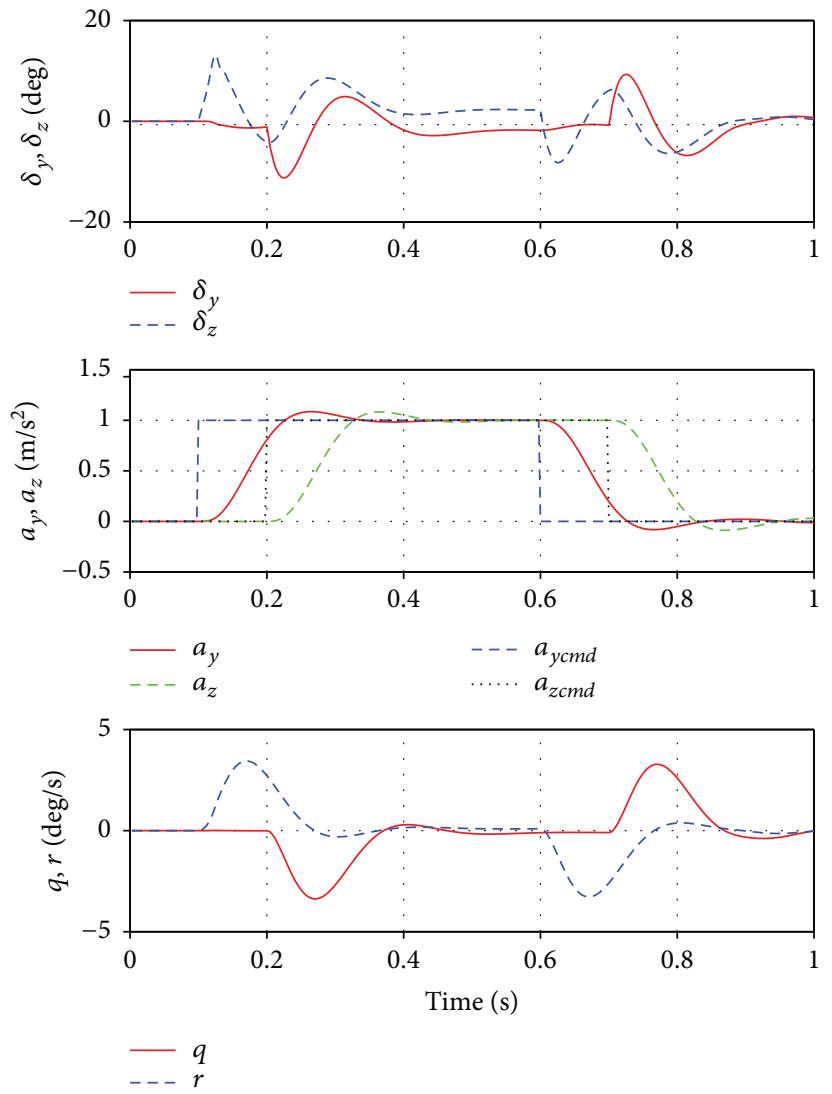

FIgURE 10: Control performance with $\Lambda=0.6 I$.

$30 \%$ from the nominal $A$ matrix) compared to those determined from wind channel tests. At this time, the eigenvalues of the system matrix have a positive real part, which means the system is unstable.

Outcomes of the pitch and yaw channel autopilots in the same set-point are presented in Figure 6. In this case, the baseline controller is not able to suppress the unstable pitch and yaw modes, whereas the adaptive controller is able. The behavior of canard angles in the pitch and yaw channels is presented in Figure 7. The amplitude of canard angles for the adaptive controller is slightly bigger than that of the nominal model, due to the uncertainty. The angular rates of pitch and yaw channels are presented in Figure 8, which implies that the adaptive controller shows a better performance.

In order to visualize the destructive effect of the control effectiveness loss, simulations with different control effectiveness are performed. According to Figures 9 and 10, the proposed adaptive controller can autoadjust the canard angles to handle the loss of control effectiveness. Considering simulation results for this scenario, the proposed adaptive controller presents a proper performance in the presence of the disturbances enforced due to the channel couplings and control effectiveness loss.

Measurement noise is also taken into account in order to carry out more investigations in the perturbed conditions. To this end, a worst-case condition is enforced; a white noise with a standard deviation of $0.25(\%)$ is injected into the rate 

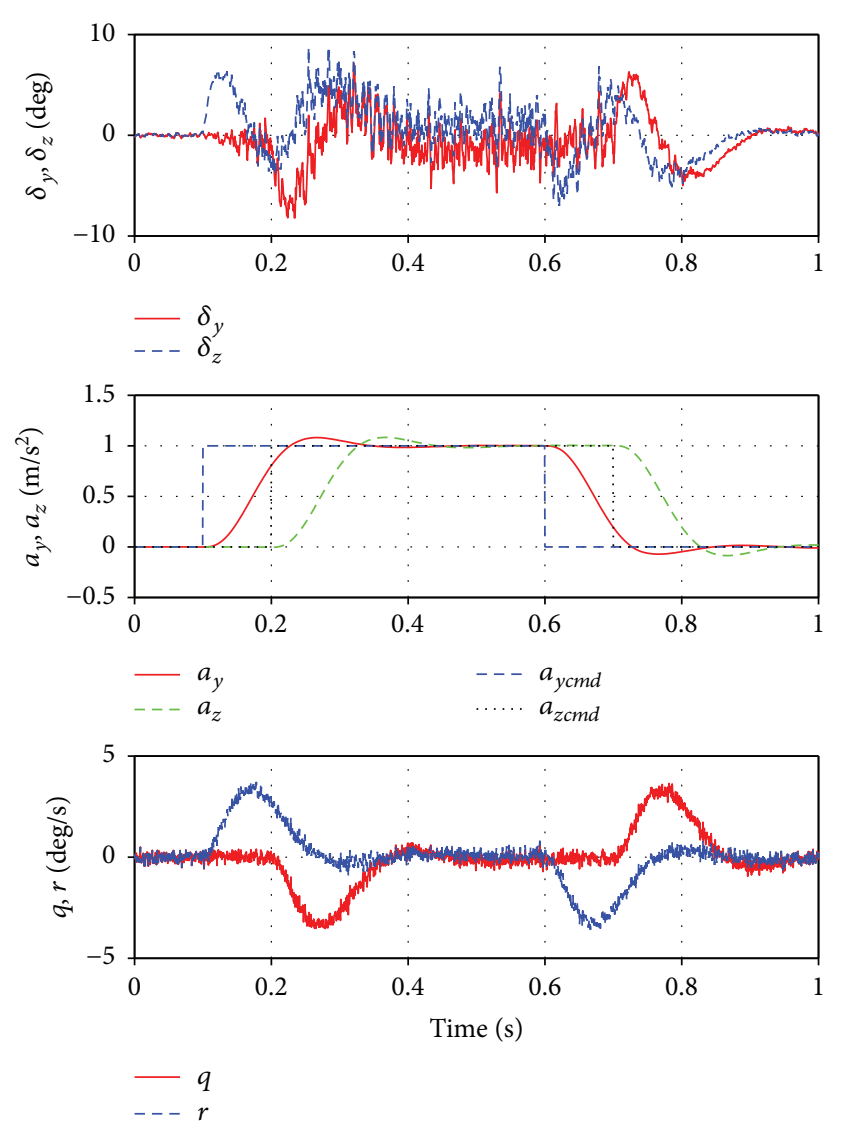

FIGURE 11: Adaptive controller performance with measurement noise.

gyroscopes of each channel, which is corresponding to the noise of low-cost MEMS gyroscopes. Figure 11 shows that the adaptive controller was robust in the presence of measurement noise in the angular rate feedback loop.

We analyze the robustness of the overall closed-loop system with the steady-state gain $K_{x}^{T}+\Psi^{T}$ in what follows. The gain and phase margin for the baseline controller and the closed-loop adaptive controller under the uncertain model are calculated as in Appendix B:

$$
\begin{aligned}
& \mathrm{GM}_{\mathrm{b} 1}=[-818.1] \mathrm{dB} \quad \mathrm{PM}_{\mathrm{b} 1}= \pm 51.9^{\circ}, \\
& \mathrm{GM}_{\mathrm{ad}}=[-14.231 .6] \mathrm{dB} \quad \mathrm{PM}_{\mathrm{ad}}= \pm 58.3^{\circ} .
\end{aligned}
$$

It can be seen that the adaptive controller, in general, is more robust than the baseline controller is.

\section{Conclusion}

This paper presents a robust adaptive output feedback autopilot for a guided spinning rocket to resistant external disturbance and maintain precision command tracking. The autopilot is composed of a baseline controller augmented with an adaptive component to accommodate control effectiveness uncertainty and matched plant uncertainty, and it makes use of the closed-loop reference model to improve the transient properties of the overall adaptive system. Adaptive control is applicable to nonsquare systems through designing mixing matrix $M$ and the observer gain matrix $L$. This procedure only needs to solve a set of linear matrix inequalities, which is given by the robust SPR lemma. The performance of the proposed adaptive output feedback controller is evaluated by numerical simulations when applied to the lateral dynamic model of a guided spinning rocket. The simulation results showed that the robust adaptive output controller can achieve asymptotic command tracking with significant uncertainty in control effectiveness, moment coefficient, and measurement noise.

\section{Appendix}

\section{A. System Matrices}

The nominal dynamical plant matrices for a guided spinning rocket of velocity $V=581 \mathrm{~m} / \mathrm{s}$, altitude $H=5000 \mathrm{~m}$, and spinning rate $p=25.4 \mathrm{rad} / \mathrm{s}$ are

$$
\begin{aligned}
A_{p} & =\left[\begin{array}{cccc}
-0.35 & 0 & 0 & -1 \\
0 & -0.35 & 1 & 0 \\
1.28 & -54.89 & -0.63 & -0.07 \\
54.89 & 1.28 & 0.07 & -0.63
\end{array}\right], \\
B_{p} & =\left[\begin{array}{cc}
0 & 0 \\
0 & 0 \\
12.15 & 0 \\
0 & 12.15
\end{array}\right], \\
C_{p} & =\left[\begin{array}{crrr}
-206.59 & 0 & 0 & 0 \\
0 & -206.59 & 0 & 0 \\
0 & 0 & 1 & 0 \\
0 & 0 & 0 & 1
\end{array}\right] \\
C_{z} & =\left[\begin{array}{crrr}
-206.59 & 0 & 0 & 0 \\
0 & -206.59 & 0 & 0
\end{array}\right]
\end{aligned}
$$

The dynamical system $\left\{A_{p}, B_{p}, C_{p}, 0\right\}$ is controllable and observable. There is no transmission zero in the plant. The linear control design parameters are given as $Q_{\mathrm{lqr}}=$ $\operatorname{diag}([0.5,0.5,0,0,0.5,0.5])$ and $R_{\mathrm{lqr}}=\operatorname{diag}([0.005,0.005])$. Following the design procedure, the baseline controller gain matrix can be computed using the MATLAB command lqr:

$$
K_{x}^{T}=\left[\begin{array}{cccccc}
0.12 & -133.98 & -4.64 & 0 & 0.02 & -10 \\
-133.98 & 0.12 & 0 & -4.64 & 10 & 0
\end{array}\right]
$$

Robust Control Toolbox 6.4 is used to solve the LMI in (36), resulting in mixing matrix $M$ and observer $L$ 


$$
\begin{aligned}
M & =\left[\begin{array}{cccccc}
0 & 0 & 0.0467 & 0 & 0 & 0 \\
0 & 0 & 0 & 0.0467 & 0 & 0
\end{array}\right], \\
L & =\left[\begin{array}{cccccc}
0 & 0 & -0.64 & -26.9 & -103.29 & 0 \\
0 & 0 & 26.9 & -0.64 & 0 & -103.29 \\
0 & -0.13 & 0 & 0 & 0 & 0 \\
0.13 & 0 & 0 & 0 & 0 & 0 \\
0.5 & 0 & 0 & 0 & -0.5 & 0 \\
0 & 0.5 & 0 & 0 & 0 & -0.5
\end{array}\right] .
\end{aligned}
$$

\section{B. Multivariable Gain and Phase Margins}

The multivariable gain and phase margin was calculated along the lines with [25], where the gain and phase margins are defined using the return difference matrix $\underline{\sigma}\left(I+L_{u}(s)\right)$ and the stability robustness matrix $\underline{\sigma}\left(I+L_{u}{ }^{-1}(s)\right)$.

The operator $\sigma($.$) corresponds to the minimum singular$ values; $L_{u}(s)$ denotes the input loop transfer function.

First, the minimum value of the return difference and the stability robustness transfer function over all frequency $\omega$ are defined as $\alpha_{\sigma}$ and $\beta_{\sigma}$, respectively:

$$
\begin{aligned}
& \alpha \sigma=\min _{\omega} \underline{\sigma}\left(I+L_{u}(s)\right), \\
& \beta \sigma=\min _{\omega} \underline{\sigma}\left(I+L_{u}{ }^{-1}(s)\right) .
\end{aligned}
$$

Then, the gain and phase margin of the return difference matrices $\mathrm{GM}_{\alpha \sigma}$ and $\mathrm{PM}_{\alpha \sigma}$ and stability robustness matrices $\mathrm{GM}_{\beta \sigma}$ and $\mathrm{PM}_{\beta \sigma}$ are calculated:

$$
\begin{aligned}
& \mathrm{GM}_{\alpha \sigma}=\left[(1+\alpha \sigma)^{-1}(1-\alpha \sigma)^{-1}\right] \\
& \mathrm{GM}_{\beta \sigma}=[1-\beta \sigma+1 \beta \sigma] \\
& \mathrm{PM}_{\alpha \sigma}= \pm 2 \sin ^{-1}\left(\frac{\alpha \sigma}{2}\right) \\
& \mathrm{PM}_{\beta \sigma}= \pm 2 \sin ^{-1}\left(\frac{\beta \sigma}{2}\right)
\end{aligned}
$$

The union of these gain and phase margins in (B.2) yields the multivariable gain margin GM and phase margin PM, which can be written compactly as

$$
\begin{aligned}
& \mathrm{GM}=\left[\mathrm{GM}_{\mathrm{L}} \mathrm{GM}_{\mathrm{U}}\right], \\
& \mathrm{PM}=\left[\mathrm{PM}_{\mathrm{L}} \mathrm{PM}_{\mathrm{U}}\right] .
\end{aligned}
$$

\section{Conflicts of Interest}

The authors declare that they have no conflicts of interest.

\section{Acknowledgments}

The grant support from the National Natural Science Foundation of China (nos. 11202023 and 11532002) is greatly acknowledged.

\section{References}

[1] J. S. Shamma and J. R. Cloutier, "Gain-scheduled missile autopilot design using linear parameter varying transformations," Journal of Guidance, Control, and Dynamics, vol. 16, no. 2, pp. 256-263, 1993.

[2] P. Apkarian, P. Gahinet, and G. Becker, "Self-scheduled $H_{\infty}$ control of linear parameter-varying systems: a design example," Automatica, vol. 31, no. 9, pp. 1251-1261, 1995.

[3] S. Theodoulis and P. Wernert, "Flight control for a class of $155 \mathrm{~mm}$ spinstabilized projectile with reciprocating canards," in AIAA Guidance, Navigation, and Control Conference, pp. 1-8, Minneapolis, MN, USA, 2012.

[4] S. Theodoulis, F. Seve, and P. Wernert, "Robust gainscheduled autopilot design for spin-stabilized projectiles with a course-correction fuze," Aerospace Science and Technology, vol. 42, pp. 477-489, 2015.

[5] X. Yan, S. Yang, and C. Zhang, "Coning motion of spinning missiles induced by the rate loop," Journal of Guidance, Control, and Dynamics, vol. 33, no. 5, pp. 1490-1499, 2010.

[6] X. Yan, S. Yang, and F. Xiong, "Stability limits of spinning missiles with attitude autopilot," Journal of Guidance, Control, and Dynamics, vol. 34, no. 1, pp. 278-283, 2011.

[7] K. Li, S. Yang, and L. Zhao, "Stability of spinning missiles with an acceleration autopilot," Journal of Guidance, Control, and Dynamics, vol. 35, no. 3, pp. 774-786, 2012.

[8] K. Li, S. Yang, and L. Zhao, "Three-loop autopilot of spinning missiles," Proceedings of the Institution of Mechanical Engineers, Part G: Journal of Aerospace Engineering, vol. 228, no. 7, pp. 1195-1201, 2014.

[9] V. Stepanyan and K. Krishnakumar, "Adaptive control with reference model modification," Journal of Guidance, Control, and Dynamics, vol. 35, no. 4, pp. 1370-1374, 2012.

[10] A. P. Nair, N. Selvaganesan, and V. R. Lalithambika, "Lyapunov based PD/PID in model reference adaptive control for satellite launch vehicle systems," Aerospace Science and Technology, vol. 51, pp. 70-77, 2016.

[11] P. Sarhadi, A. R. Noei, and A. Khosravi, "Adaptive integral feedback controller for pitch and yaw channels of an AUV with actuator saturations," ISA Transactions, vol. 65, pp. 284-295, 2016.

[12] Z. Shi and L. Zhao, "Robust model reference adaptive control based on linear matrix inequality," Aerospace Science and Technology, vol. 66, pp. 152-159, 2017.

[13] S. Li and G. Tao, "Output feedback MIMO MRAC schemes with sensor uncertainty compensation," in Proceedings of the American Control Conference, pp. 3229-3234, Baltimore, MD, USA, 2010.

[14] J. M. Selfridge and G. Tao, "Multivariable output feedback MRAC for a quadrotor UAV," in Proceedings of the American Control Conference, pp. 492-499, Boston, MA, USA, 2016.

[15] Z. Shi and L. Zhao, "Adaptive output feedback autopilot design for spinning projectiles," in Chinese Control Conference, pp. 3516-3521, Dalian, China, 2017.

[16] D. d. S. Madeira and J. Adamy, "On the equivalence between strict positive realness and strict passivity of linear systems," IEEE Transactions on Automatic Control, vol. 61, no. 10, pp. 3091-3095, 2016.

[17] C. H. Huang, P. A. Ioannou, J. Maroulas, and M. G. Safonov, "Design of strictly positive real systems using constant output 
feedback," IEEE Transactions on Automatic Control, vol. 44, no. 3, pp. 569-573, 1999.

[18] I. Barkana, "Comments on "Design of strictly positive real systems using constant output feedback"," IEEE Transactions on Automatic Control, vol. 49, no. 11, pp. 2091-2093, 2004.

[19] R. Johansson and A. Robertsson, "Observer-based strict positive real (SPR) feedback control system design," Automatica, vol. 38, no. 9, pp. 1557-1564, 2002.

[20] A. Saberi and P. Sannuti, "Squaring down by static and dynamic compensators," IEEE Transactions on Automatic Control, vol. 33, no. 4, pp. 358-365, 1988.

[21] A. Fradkov, "Passification of non-square linear systems and feedback Yakubovich-Kalman-Popov lemma," European Journal of Control, vol. 9, no. 6, pp. 577-586, 2003.

[22] C. H. Murphy, Free Flight Motion of Symmetric Missiles, Technical Report, U.S.Army Ballistic Research Laboratories, Aberdeen Proving Ground, Maryland, 1963.

[23] M. Pechier, P. Guillen, and R. Cayzac, "Magnus effect over finned projectiles," Journal of Spacecraft and Rockets, vol. 38, no. 4, pp. 542-549, 2001.

[24] L. Zhao, Z. Shi, and Y. Zhu, "Acceleration autopilot for a guided spinning rocket via adaptive output feedback," Aerospace Science and Technology, vol. 77, pp. 573-584, 2018.

[25] E. Lavretsky and K. A. Wise, "Robust and Adaptive Control with Aerospace Applications," in Advanced Textbooks in Control and Signal Processing, Springer, London, UK, 2013.

[26] D. P. Wiese, A. M. Annaswamy, J. A. Muse, M. A. Bolender, and E. Lavretsky, "Adaptive output feedback based on closed-loop reference models for hypersonic vehicles," Journal of Guidance, Control, and Dynamics, vol. 38, no. 12, pp. 24292440, 2015.

[27] T. E. Gibson, Z. Qu, A. M. Annaswamy, and E. Lavretsky, "Adaptive output feedback based on closed-loop reference models," IEEE Transactions on Automatic Control, vol. 60, no. 10, pp. 2728-2733, 2015.

[28] Z. Qu and A. M. Annaswamy, "Adaptive output-feedback control with closed-loop reference models for very flexible aircraft," Journal of Guidance, Control, and Dynamics, vol. 39, no. 4, pp. 873-888, 2016.

[29] B. Brogliato, B. Maschke, R. Lozano, and O. Egeland, Dissipative Systems Analysis and Control, Theory and Applications, Springer Verlag, London, UK, 2nd edition, 2007.

[30] L. Xie, M. Fu, and C. E. de Souza, " $H_{\infty}$ infinity control and quadratic stabilization of systems with parameter uncertainty via output feedback," IEEE Transactions on Automatic Control, vol. 37, no. 8, pp. 1253-1256, 1992.

[31] I. Barkana, "The new theorem of stability-direct extension of lyapunov theorem," Mathematics in Engineering, Science \& Aerospace (MESA), vol. 6, no. 3, pp. 519-550, 2015.

[32] I. Barkana, "Barbalat's lemma and stability-misuse of a correct mathematical result?," Mathematics in Engineering, Science \& Aerospace (MESA), vol. 7, no. 1, pp. 197-219, 2016. 


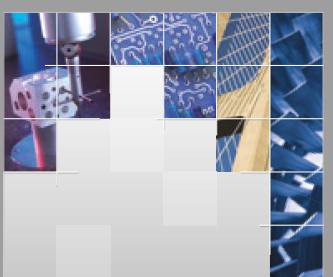

\section{Enfincering}
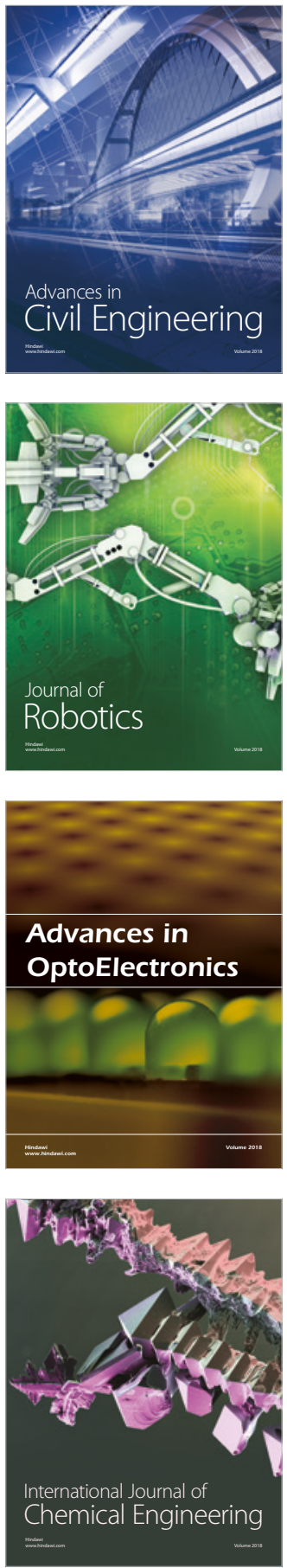

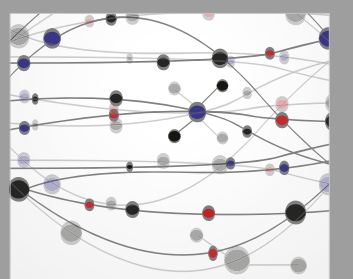

\section{Rotating \\ Machinery}

The Scientific World Journal

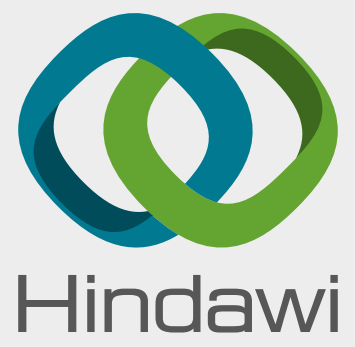

Submit your manuscripts at

www.hindawi.com
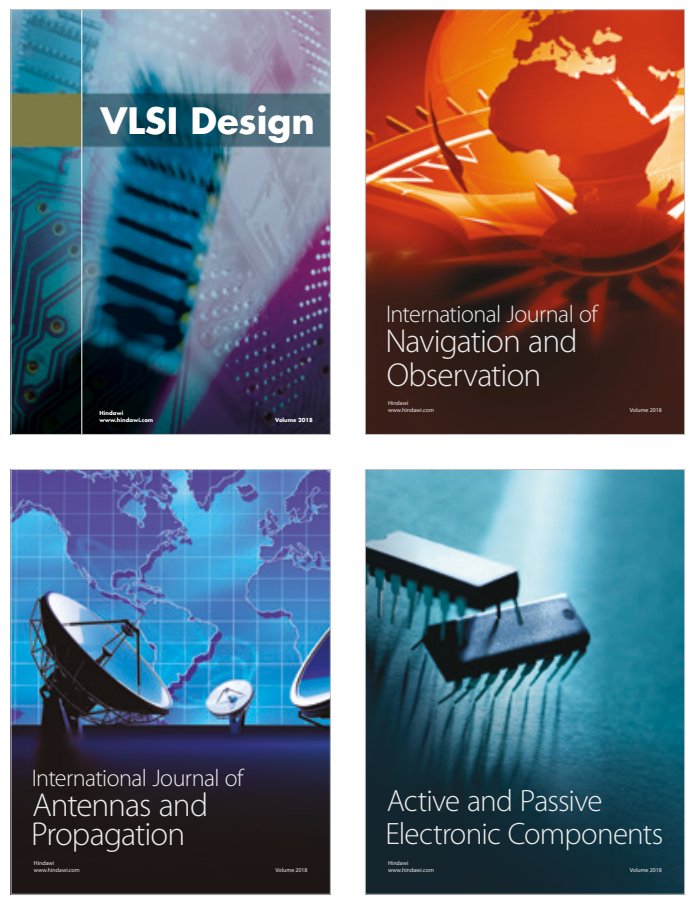
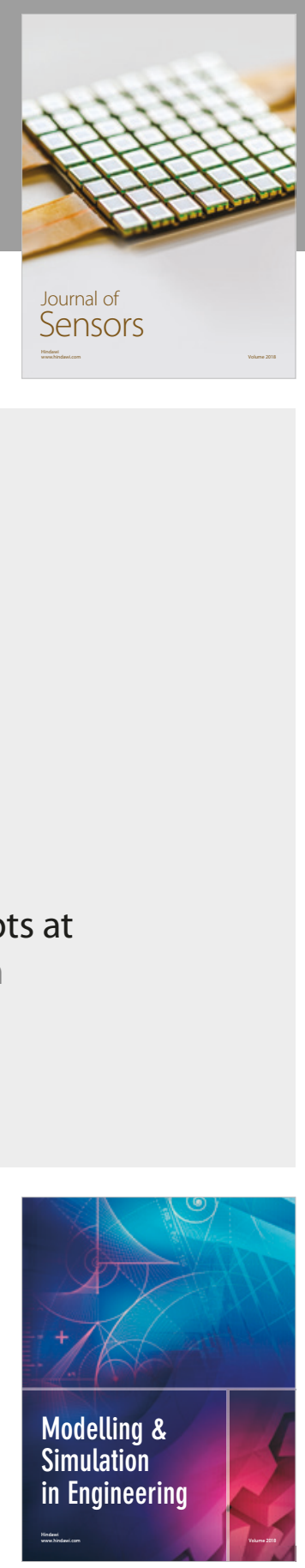

\section{Advances \\ Multimedia}
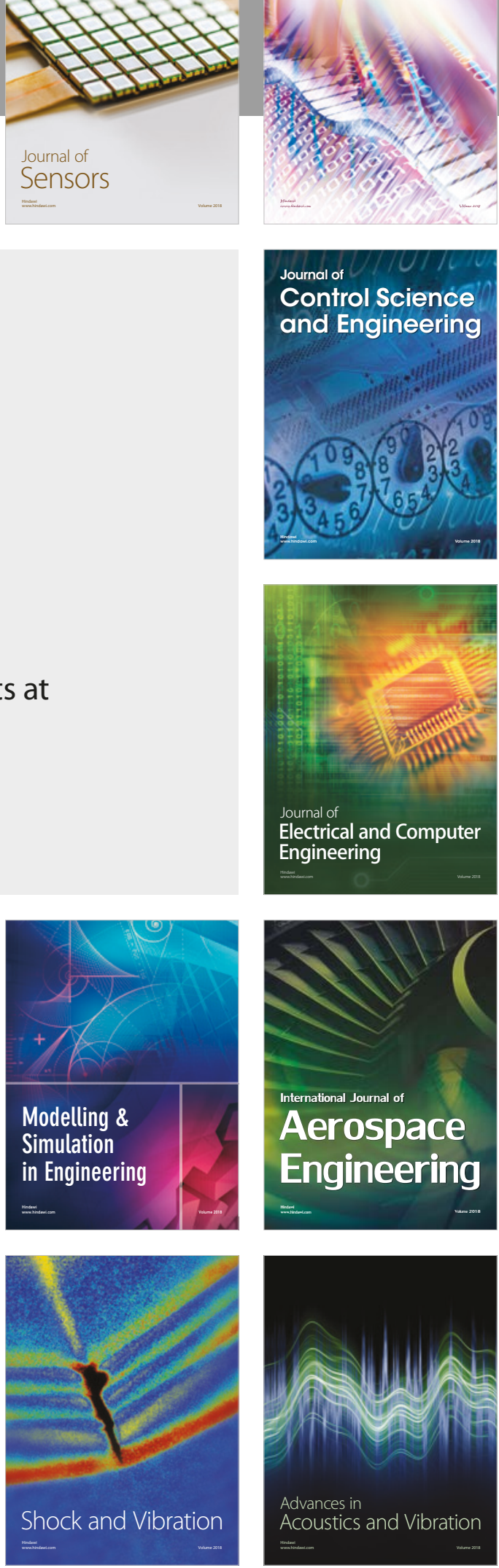